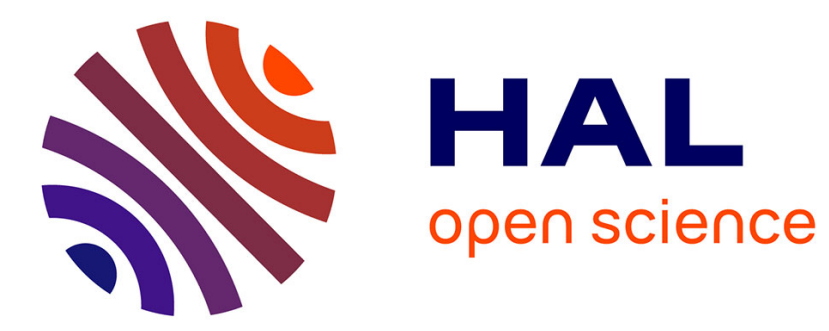

\title{
A quantitative measure of migratory connectivity
}

Roberto Ambrosini, Anders Pape Møller, Nicola Saino

\section{To cite this version:}

Roberto Ambrosini, Anders Pape Møller, Nicola Saino. A quantitative measure of migratory connectivity. Journal of Theoretical Biology, 2009, 257 (2), pp.203. 10.1016/j.jtbi.2008.11.019 . hal00554539

\section{HAL Id: hal-00554539 \\ https://hal.science/hal-00554539}

Submitted on 11 Jan 2011

HAL is a multi-disciplinary open access archive for the deposit and dissemination of scientific research documents, whether they are published or not. The documents may come from teaching and research institutions in France or abroad, or from public or private research centers.
L'archive ouverte pluridisciplinaire $\mathbf{H A L}$, est destinée au dépôt et à la diffusion de documents scientifiques de niveau recherche, publiés ou non, émanant des établissements d'enseignement et de recherche français ou étrangers, des laboratoires publics ou privés. 


\section{Author's Accepted Manuscript}

A quantitative measure of migratory connectivity

Roberto Ambrosini, Anders Pape Møller, Nicola

Saino

PII: $\quad$ S0022-5193(08)00616-4

DOI: $\quad$ doi:10.1016/j.jtbi.2008.11.019

Reference: $\quad$ YJTBI5381

To appear in: $\quad$ Journal of Theoretical Biology

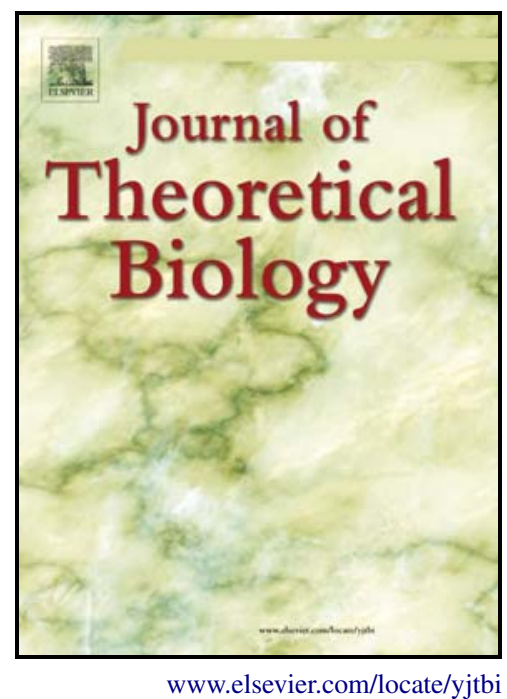

Received date: $\quad 12$ February 2008

Revised date: $\quad 18$ October 2008

Accepted date: 26 November 2008

Cite this article as: Roberto Ambrosini, Anders Pape Møller and Nicola Saino, A quantitative measure of migratory connectivity, Journal of Theoretical Biology (2008), doi:10.1016/j.jtbi.2008.11.019

This is a PDF file of an unedited manuscript that has been accepted for publication. As a service to our customers we are providing this early version of the manuscript. The manuscript will undergo copyediting, typesetting, and review of the resulting galley proof before it is published in its final citable form. Please note that during the production process errors may be discovered which could affect the content, and all legal disclaimers that apply to the journal pertain. 


\section{A quantitative measure of migratory}

\section{connectivity}

Roberto Ambrosini ${ }^{a}$, Anders Pape Møller ${ }^{b}$ and Nicola Saino ${ }^{c}$

${ }^{a}$ Dipartimento di Biotecnologie e Bioscienze, Università degli Studi di Milano Bicocca, piazza della Scienza 2, I-20126 Milano, Italy. E-mail: roberto.ambrosini@unimib.it

$9{ }^{\mathrm{b}}$ Laboratoire de Parasitologie Evolutive, CNRS UMR 7625, Université Pierre et Marie Curie, 7 quai St. Bernard, Case 237, F-75252 Paris Cedex 05, France. E-mail: amoller@snv.jussieu.fr via Celoria 26, I-20133 Milano, Italy. E-mail: nicola.saino@unimi.it

16 Roberto Ambrosini, Dipartimento di Biotecnologie e Bioscienze, piazza della Scienza 2, 


\section{Abstract}

24 The study of the extent of the connection between areas where populations of birds breed

25 and areas where they winter has flourished in recent years mainly thanks to the

26 development of new techniques, but also due to traditional ringing and recovery schemes,

27 which allow tracking of individuals or populations linking wintering and breeding

28 distributions. Currently, studies on migratory connectivity focus on retention of breeding

29 population spatial structure on the non-breeding grounds and vice versa.

30 Here we propose a method to quantify migratory connectivity based on Mantel

31 correlation coefficients and to statistically test for deviations of the observed connectivity

32 from a random mix of individuals. In addition, we propose a procedure, based on

33 clustering algorithms, to identify whether observed connectivity depends on aggregation

34 of individuals or on rigid transference of distribution patterns between areas.

35 We applied this method to a large dataset of ringing recoveries of barn swallows

36 (Hirundo rustica L) migrating from their Western Palearctic breeding areas to sub-

37 Saharan winter quarters. We show that migration of barn swallow populations connects

38 specific breeding and wintering areas, and that the 'sub-populations' quantitatively

39 identified by our method are consistent with qualitative patterns of migratory connectivity

40 identified by studies of individual geographical populations based on other methods.

41 Finally, we tested the performance of the method by running simulations under different

42 scenarios. Such simulations showed that the method is robust and able to correctly detect

43 migratory connectivity even with smaller datasets and when a strong geographical pattern

44 is not present in the population. Our method provides a quantitative measure of migratory 
45 connectivity and allows for the identification of populations showing high connectivity

46 between the breeding and wintering areas. This method is suitable for a generalized

47 application to diverse animal taxa as well as to large scale analyses of connectivity for 48 conservation purposes.

49

50

51 Keywords: barn swallow; Hirundo rustica; k-means clustering; Mantel test; migration; 52

53 


\section{Introduction}

55 Migratory connectivity has been defined as the links between breeding and non-breeding

56 areas of migratory animals due to migration (Webster et al 2002). Several different

57 approaches have been proposed for the study of migratory connectivity, such as ringing

58 recoveries (Anderson et al 2000), satellite radio telemetry (Cohn 1999), stable isotope

59 analysis of feathers (e.g. Hobson and Wassenaar 1997; Marra et al 1998; Møller and

60 Hobson 2003; Hobson 2005), genetic analysis (Webster et al 2002; Lopes et al 2006), and

61 correlation analysis between point estimates of annual survival rates and proxies of

62 ecological conditions putatively affecting survival (e.g. the Normalised Difference

63 Vegetation Index; Szép et al 2006).

64 A qualitative measure of migratory connectivity has been proposed by Webster et

65 al (2002), who argued that "strong" connectivity occurs when most individuals from one

66 breeding population move to the same non-breeding location, while a "weak" or "diffuse"

67 connectivity occurs when individuals from a single breeding population spread through

68 several non-breeding grounds. Statistical approaches that have been proposed to quantify

69 migratory connectivity are based on the same procedure that is commonly used to solve

70 the problem of the "derivation of harvest" in waterfowl management (Marra et al 2006).

71 They imply the definition of transition probabilities that describe how individuals from a

72 breeding population move to each wintering location and the calculation of conditional

73 probabilities of derivation of an individual from a given site ("origin probabilities") using

74 Bayes' Rule (Marra et al 2006). This procedure, however, requires an a priori

75 identification of 'sub-ranges' (see Webster et al 2002; Marra et al 2006), which may be a 
76 difficult and somewhat arbitrary task, at least in species with continuous breeding and/or

77 non-breeding ranges. In addition, estimating conditional probabilities in such Bayesian

78 framework requires prior knowledge of population sizes, at least relative to each other,

79 among the various origin sub-ranges (Marra et al 2006) that may be difficult to acquire

80 unless information from intensive, large-scale population survey programs is available.

81 Finally, recovery probabilities must be assumed to be proportional to the relative

82 abundance on the origin grounds. If this assumption is unwarranted, true connectivity

83 among sub-populations may be overlooked.

84 Currently, studies on migratory connectivity focus on the retention of breeding

85 population structure on the non-breeding grounds (and vice versa) (Marra et al 2006). If

86 individuals that breed close to each other also winter close to each other (allohiemy sensu

87 Salomonsen 1955) then a strong connectivity occurs, while if they mix (sinhiemy)

88 connectivity is weak. Theoretically, if the positions of individuals, both in the breeding

89 and the wintering grounds are known, then two distance matrices can be calculated,

90 representing the distance within each pair of individuals in the breeding or the wintering

91 grounds, respectively. Besag and Diggle (1977) proposed to test for the significance of

92 the maintenance of reciprocal distribution in breeding and wintering grounds in a

93 blackbird (Turdus merula $\mathrm{L}$ ) population by testing for the significance of the correlation

94 between the matrix of distances in the breeding grounds and that in the wintering

95 grounds. If individuals that breed close together also winter close together (i.e. the

96 population shows strong connectivity), then a strong positive correlation is expected

97 between the two matrices. Conversely, if individuals mix (weak connectivity) a weak

98 correlation is expected. A measure of the correlation between the two distance matrices 
99 can therefore be used to quantify migratory connectivity and to statistically test for the

100 probability of observing a given pattern, under the null hypothesis of a random mix

101 among individuals at the breeding and/or the non-breeding grounds, a condition for which

102 a correlation coefficient of zero is expected. Such a test, known as the Mantel test (Sokal

103 and Rohlf 1995), does not imply an a priori identification of separated sub-ranges in the

104 breeding and wintering grounds. We emphasize that, in this approach, the length of the

105 migration journey is not taken into account as the only measures involved in the analysis

106 are distances between individuals in the breeding and in the wintering grounds.

107 A significant correlation between the distance matrices, however, can result from

108 two different processes. The first consists of individuals that maintain the same reciprocal

109 position in the breeding and wintering grounds. Following Besag and Diggle (1977) we

110 refer to this process as to "distribution pattern transfer". We note that the correlation

111 between the distance matrices will not vary under translation, rotation and scale change

112 of the pattern of distribution of individuals. Alternatively, if the population under study

113 consists of groups of individuals that stay close together both in the breeding and in the

114 wintering grounds, then a significant correlation between the distance matrices is also

115 expected, as within-group distances will, on average, be smaller than distances between

116 individuals of different groups.

117 A procedure for the quantitative study of migratory connectivity should ideally

118 allow disentangling these two processes and, if necessary, to identify the clusters of

119 individuals that breed and winter together. These goals can be achieved by applying a

120 clustering algorithm to a distance matrix obtained from the combination of the two

121 matrices of distances between individuals at the breeding grounds and in the winter 
122 quarters. A significant connectivity in a non-structured (i.e. non-clustered) population

123 indicates that migratory connectivity arises from a process of distribution pattern

124 transference. Conversely, if cluster analysis reveals a structure in the population, then

125 grouping of individuals that always stay together contributes to migratory connectivity.

126 Within (main) clusters, individuals may then show pattern transference or further

127 grouping, resulting in sub-clusters.

128 In this study, we thus propose a novel approach to quantify migratory

129 connectivity. The method is based on Mantel test to assess the strength of migratory

130 connectivity and cluster analysis to investigate the process that generates it and to unravel

131 the clustered structure of the population.

132 We first assessed the performances of the method by running simulations under

133 three different scenarios. In the first scenario, the connectivity was due to a process of

134 distribution pattern transference. In the second, connectivity arose from the clustered

135 structure of the simulated population, but no pattern transference occurred for the

136 individuals of the same cluster. In the third, we simulate a condition where both processes

137 occur simultaneously. In addition, to exemplify the use of this method, we apply it to a

138 large dataset of recoveries of barn swallows (Hirundo rustica $\mathrm{L}$ ) ringed in their breeding

139 range in Europe and recovered in their sub-Saharan wintering quarters or vice versa.

140 Data from recoveries of ringed birds as a tool in the study of connectivity have

141 been criticized on several bases, such as the large biases in the probability of recovery of

142 individuals between different parts of the world (Webster et al 2002). Still, they represent

143 the largest (and largely under-utilised) datasets currently available on numerous

144 migratory species. The results obtained from the analysis of the present dataset should 
145 therefore be considered with this caveat in mind, and they will be compared to other

146 available information about the migration of barn swallows, including stability of the

147 genetic variance-covariance matrix of different populations (Roff et al 2004) and stable

148 isotope analyses of feathers of different populations (Evans et al 2003; Møller and

149 Hobson 2003).

150 The barn swallow is a small passerine bird that migrates each year from Europe to

151 Africa south of the Sahara desert. A considerable effort has been spent in the last decades

152 to capture and ring barn swallows both in Africa and Europe mainly within the EURING

153 and SAFRING ringing schemes. Recoveries and recaptures of barn swallows are

154 available from the entire breeding and wintering range of the species, encompassing an

155 extremely wide geographical area and a very long time span. To the best of our

156 knowledge, this is one of the largest available data sets of ringing recoveries and we are

157 convinced that the amount of information it contains largely overcomes the 'noise' due to

158 differences in ringing and capture efforts in different locations and years. 
162 The method we are proposing is based on the coordinates of individuals recorded in the

163 areas occupied in different phases of the annual cycle. We assume that such grounds are

164 geographically separated and individuals move annually between them during migrations.

165 Since the large majority of the data about migrants come from ornithological studies, we

166 will refer to these areas as the breeding and the wintering grounds, albeit these definitions

167 may not be strictly applicable to other migrants (e.g. whales, for which 'breeding' and

168 'feeding' grounds may be more appropriate). In all the analysis, including the

169 simulations, we always started from datasets where the position of individuals was

170 expressed by geographical coordinates, since in real datasets position of individuals is

171 usually recorded in this way. Nevertheless, this method is applicable to any other

172 coordinate system as it basically requires only distances between individuals.

173

174 Analysis of migratory connectivity

175

176 From geographical coordinates of individuals both in the breeding and wintering grounds,

177 we calculated two matrices of orthodromic distances (i.e. the minimum path on the

178 surface of the Earth), between all pairs of barn swallows both in the breeding and the

179 wintering quarters (see Fig. 1 for further details).

180 Mantel correlation coefficient $\left(r_{M}\right)$ was used to test for the correlation between

181 orthodromic distance matrices. It corresponds to a simple Pearson product moment

182 correlation coefficient between the two matrices, whose significance is assessed with a 
183 randomization procedure because distances between individuals within each distance

184 matrix are not statistically independent (Sokal and Rohlf 1995). We randomly permutated

185 the position of individuals at the breeding grounds 999 times; for each permutation, a

186 distance matrix was calculated and its correlation coefficient with the actual distance

187 matrix of individuals at the wintering grounds was calculated. The significance of the

188 observed Mantel coefficient was determined based on its rank in the set of the

189 coefficients from the randomization procedure as $P=[(1000-$ rank $) / 1000]$.

190 The analysis of the spatial structure of the dataset was based on a cluster detection

191 approach. First, we combined the two distance matrices into one by computing, for each

192 pair of individuals, the square root of the sum of their squared orthodromic distances at

193 the breeding and wintering grounds. This measure can be considered an overall distance

194 index similar to the Euclidean distance between two points that also takes the round

195 shape of the Earth into account as it is based on orthodromic distances.

196 Cluster detection was then performed with the pam algorithm in S-Plus 4.5

197 applied to the overall distance indices matrix. The number of clusters was identified as

198 the number that maximized the overall average silhouette width (hereafter oasw for

199 brevity) (Rousseeuw 1987). The pam procedure (fully described in Kaufman and

200 Rousseeuw 1990) is a partitioning clustering algorithm where the number of clusters

201 must be indicated a priori. The oasw is a measure of the goodness of the overall

202 classification of points in a given number of clusters. It is a dimensionless coefficient of

203 the extent of structure of clustering that has been identified. It is defined as the average

204 value of the silhouettes s(i) computed over all objects in the dataset where 


$$
\mathrm{s}(i)=\frac{\mathrm{b}(i)-\mathrm{a}(i)}{\max \{\mathrm{a}(i) ; \mathrm{b}(i)\}}
$$

208 a $(i)$ being the average dissimilarity of $i$ to all other objects of the cluster to which $i$

209 belongs and $\mathrm{b}(i)$ the average dissimilarity of $i$ to all objects of the nearest cluster to which

$210 i$ does not belong. $\mathrm{s}(i)$ values range between -1 and 1 and the larger the value the better

211 the classification of an object in a given cluster (Rousseeuw 1987). The best number of

212 clusters corresponds to the number that maximizes the oasw. Oasw values lower than

2130.25 indicate that no substantial structure has been found in the data, values between 0.26

214 and 0.50 indicate a "weak" structure, between 0.51 and 0.70 a "reasonable" structure and

215 values larger than 0.71 a "strong" structure (Rousseeuw 1987). Each cluster detected in

216 the first step was investigated further with the same procedure, and, if a significant

217 structure was recognized, it was divided into sub-clusters, numbered as (dataset).(main

218 cluster).(sub-cluster).

220 Simulations

222 In all simulations we assumed a uniform spherical surface with no limits to the movement

223 of individuals. The centre of the cluster(s) of individuals in the breeding grounds were

224 always set at $30^{\circ} \mathrm{N}$, while at $30^{\circ} \mathrm{S}$ in the wintering grounds. Longitude of the cluster

225 centre(s) varied during simulations (see below). Positions of individuals were generated

226 as a random determination from a bivariate Gaussian distribution with unit standard

227 deviation (SD) centred on the centre(s) of the cluster(s). The distance between cluster

228 centres and the random noise added to individual positions (see below) were expressed as 
229 standard deviations (SD) of the bivariate Gaussian used to generate individual position

230 within clusters. At each step of the simulation, all the analyses were run according to the

231 method described above, and the significance of the Mantel correlation coefficient, the

232 oasw value, the number of clusters identified and the proportion of individuals correctly

233 classified were recorded.

234 The first simulation aimed at testing the ability of the method we propose to

235 detect migratory connectivity arising from a process of distribution pattern transference

236 (Besag and Diggle 1977). One cluster with 200 individuals was generated in the breeding

237 ground. In a process of pure pattern transference, the reciprocal position of individuals in

238 the wintering grounds should strictly reflect that in the breeding grounds except for

239 random variation. This process was simulated by generating positions in the wintering

240 grounds corresponding to those in the breeding grounds plus a bivariate normal random

241 noise added to the position of each individual. At each step of the simulation, the standard

242 deviation of the noise increased by $0.1 \mathrm{SD}$ from 0 to $4 \mathrm{SD}$. Hence, this simulation was

243 run 41 times.

244 The second simulation aimed at testing the ability of the method to detect

245 migratory connectivity arising from a clustered structure in the population. Two clusters

246 of 100 individuals were generated. The position of individuals within each cluster both in

247 the breeding and in the wintering grounds was randomly determined. Hence, no pattern

248 transference existed within each cluster. At each run of the simulation, the distance

249 between cluster centres in the wintering and/or in the breeding grounds increased by $1 \mathrm{SD}$

250 from 0 (i.e. clusters superimposed both in the breeding and in the wintering grounds) to

$25110 \mathrm{SD}$, so that this simulation was run 121 times. 
The third simulation investigated a situation when both clustering and pattern

253 transference occurred. This third simulation followed the general scheme of the second,

254 involving two clusters of individuals with increasing distance between cluster centres,

255 but, as in the first simulation, the reciprocal position of individuals within clusters in the

256 wintering grounds reflected that in the breeding grounds plus a random Gaussian noise

257 with $\mathrm{SD}=0.2$. This value was arbitrarily chosen as the first simulation demonstrated that

258 the amount of noise does not influence the performance of the method (see below).

260 The barn swallow dataset

262 The whole dataset consisted of recoveries of individually marked barn swallows between

2631911 and 1998. Only first recoveries were selected in order to avoid pseudo-replication.

264 Individuals breeding east of longitude $60^{\circ} \mathrm{E}$ (approximately corresponding to the Ural

265 Mountains) were excluded. A first dataset (hereafter called "All” for brevity) of 1103

266 barn swallows included all individuals that were within the breeding range of the species

267 between April and September and within the wintering range between October and

268 March. We then quantitatively assessed the potential noise arising from recoveries of

269 individuals during migration by selecting a second dataset ("FP") of 225 barn swallows

270 that were within the breeding range in May-June and within the winter quarters in

271 December-February, i.e. in the focal periods of reproduction and wintering, respectively.

272 Natal dispersal in the barn swallow is larger than breeding dispersal (Turner 1994). This

273 may bias the results if the proportion of ringed adults and young varies geographically. In

274 addition, in Africa a large number of barn swallows was ringed in a rather small number 
275 of sites so that cluster detection could be biased by the small distance between ringing

276 sites of many individuals. Hence, a third dataset (“AE”) was selected, only including barn

277 swallows ringed in Europe as adults. Finally, the two previous selection criteria were

278 combined to obtain a fourth restricted dataset ("R") of 71 barn swallows ringed as adults

279 in Europe in May-June and recovered in Africa in December-February. We then

280 compared the results of the analyses based on the four datasets by investigating

281 consistency of classification of individuals into clusters.

282

283 


\section{Results}

\section{Simulations}

285 In all the 41 runs of the first simulations, a highly significant Mantel correlation

286 coefficient was found $\left(r_{M}<0.001\right.$ in all cases $)$. No cluster structure was present in the

287 data and therefore classification of individuals into clusters was not relevant.

288 Fig. 2 summarizes the results of the second simulation. In 14 cases, the method

289 failed to detect connectivity, i.e. both the Mantel correlation coefficient was not

290 significant and the oasw value was lower than 0.5. In all these cases, the distance between

291 cluster centres in one area was 0 or 1 SD. Clusters generated as random determination

292 from a bivariate Gaussian are roughly circular and about 90\% of individuals lie within 2

293 SD from the centre. Hence, a distance of 1 SD between cluster centres implies that at

294 least $60 \%$ of individuals lie in the common area. We note that no within-cluster pattern

295 transference occurred in this simulation, so that non-significant Mantel coefficients were

296 expected when clusters were close together. In another 14 cases the oasw value did not

297 indicate a reasonable structure in the data, but the Mantel coefficient was significant. In

298 all these cases the two clusters partly overlapped at least in one area. However, also in

299 these cases more than $90 \%$ of individuals were correctly classified in two clusters.

300 Mantel correlation coefficient was significant in all the 121 runs of the third

301 simulation. In 24 cases, always with partly overlapping clusters, oasw values did not

302 reveal a reasonable structure in the data albeit only in 10 cases cluster analysis correctly

303 classified less than $90 \%$ of individuals.

305 Barn swallow dataset 
306 The Mantel correlation coefficient for the "All" dataset was 0.0247 (Fig. 3a). In the

307 randomization procedure we obtained 54 times a correlation coefficient larger than the

308 observed one. Migratory connectivity should therefore be considered marginally non-

309 significant $(P=0.054)$. However, the oasw value suggested a "reasonable" structure with

310 two clusters that also emerged from the analysis of the data subsets (see below). For this

311 reason, we identified two clusters that are shown in Fig. 4a and pictorially in Fig. 5a. The

312 corresponding silhouette plot is shown in Fig. 6. Mean orthodromic distance between

313 individuals in Europe was 1358.16 (941.673 SD) km (range 0-5400.34 km) while in

314 Africa it was 1493.67 (1553.880 SD) km (range 0-6192.57 km). Hence, distances in both

315 ranges were similar, and the pattern of distribution in the two ranges had similar effects

316 on cluster detection and composition. Cluster All.1 mainly included barn swallows

317 breeding in South-Western Europe and wintering from Liberia to Uganda, while cluster

318 All.2 was mainly constituted of barn swallows from Northern Europe that winter south of

319 the Equator, from Zaire to South Africa. The clusters partly overlap at the breeding

320 grounds while they are well separated in the wintering grounds.

321 Mantel tests showed a significant migratory connectivity for cluster All.2 and a

322 marginally non-significant connectivity for cluster All.1 that, however, showed an oasw

323 of 0.433 (i.e. reasonably high) (Fig. 3). We therefore analyzed the structure of both

324 clusters (see also Discussion) that could be divided into two sub-clusters each (All.1.1,

325 All.1.2, All.2.1 and All.2.2, pictorially shown in Fig. 5b). In the next step of the analysis,

326 only clusters All.1.2 and All.2.1 could be further divided in two and six third-level sub-

327 clusters, respectively. However, the results at this fine level were inconsistent between

328 data subsets (see below), and they are therefore not presented in detail. 
The "FP" subset showed a significant connectivity $\left(n=255, r_{M}=0.0701, P=\right.$

330 0.005). The oasw reached its maximum value $(=0.582)$ for two clusters, thus indicating a

331 reasonable structure in the data. At the second step of analysis, cluster FP.1 could be

332 divided into two second-level sub-clusters based on oasw value $\left(n=32, r_{M}=0.0768, P=\right.$

3330.140$, oasw $=0.484)$, while cluster FP.2 was significantly structured with two sub-

334 clusters $\left(n=223, r_{M}=0.0670, P=0.002\right.$, oasw $\left.=0.510\right)$, none of which could be further

335 subdivided (details not shown). These results are consistent with the results of the

336 analysis of the "All" set of data. Indeed, all individuals classified in cluster FP.1 had been

337 classified in cluster All.1 and all individuals in FP.2 had been classified in All.2. At the

338 second level of analysis, only 3 individuals were misclassified.

339 The details of the results of the analysis of the "AE" set of barn swallows are

340 shown in Fig. 3b. The individuals showed significant connectivity and could be grouped

341 into two clusters that, in turn, were both structured in two sub-clusters. Sub-clusters

342 AE.1.1 and AE.1.2 showed non-significant connectivity, while AE.2.1 and AE.2.2 were

343 structured and could be divided into two and four third-level sub-clusters, respectively

344 (details not shown). However, as we did for the "All" dataset, we cautiously considered

345 the results only to the second step of the analysis. The results obtained from this selection

346 criterion were highly consistent with the results of the analysis of the "All" dataset at the

347 first level of analysis, and at the second level for cluster AE.1. Indeed only 3 out of the 86

348 individuals classified in AE.1 were classified in All.2, and 3 individuals of AE.1.2 were

349 classified in All.2.1. The partitioning of cluster AE.2 was not consistent with that

350 obtained from the "All" dataset. This was due to the small number of barn swallows (32)

351 in cluster All.2.1 that were ringed in Europe as adults and thus included in the "AE" 
352 subset.

353 The most restricted subset of data ("R") only included 71 barn swallows. The

354 Mantel test indicated a significant connectivity $\left(r_{M}=0.2243, P<0.001\right)$, and the oasw

355 value $(=0.646)$ showed that it could be divided in two sub-clusters. The first cluster

356 could be divided in two sub-clusters according to oasw value (R.1: $n=32, r_{M}=0.0768, P$

$357=0.155$, oasw $=0.484)$ while the second was not structured (R.2: $n=39, r_{M}=-0.0371, P$

$358=0.555$, oasw $\leq 0.367)$. All individuals in R.1 had previously been classified in All.1 and

359 all individuals in R.2 in All.2. Consistent results emerged also at the second level with no

360 individual being misclassified.

361 Thus, the four levels of selection of the dataset, despite differing in composition

362 and number of individuals, led to highly consistent clustering of individual barn

363 swallows, whereby the large majority of individuals were assigned to corresponding

364 clusters in the different analyses.

365 These largely consistent results could arise because of the geographical structure

366 that the barn swallow population showed in the winter quarters, with two latitudinally

367 well-separated main clusters. To further investigate the robustness and the generality of

368 our method we then re-ran the first level analysis on a partly simulated dataset. We

369 assigned to barn swallows classified in cluster All.2 a new position in the wintering

370 ground by adding to their actual latitude the difference in latitude between the centres of

371 clusters All.1 and All.2, while leaving unchanged longitude in the wintering grounds as

372 well as position in the breeding area (see Fig. 5). This partly de-structured our dataset.

373 Mantel correlation coefficient for this partly simulated dataset increased to $0.0519, P=$

3740.002 . The cluster algorithm identified three rather than two first level clusters (oasw $=$ 
375 0.5067), which however corresponded to cluster All.1, All.2.1 and All.2.2, respectively, 376 with only 31 out of 1103 individuals being misclassified. Hence, despite the weaker

377 geographical structure, our method was able to detect the cluster structure present in the 378 data.

379 
381 In this study, we propose a novel approach to quantitatively and objectively investigate

382 migratory connectivity, which can be applied to any organism where clearly distinct

383 geographical ranges, corresponding to different phases of the life cycle, can be identified.

384 We also propose a method to quantify migratory connectivity based on the Mantel

385 correlation coefficient and a method to distinguish between two processes that can

386 generate the observed connectivity, i.e. distribution pattern transfer versus clustering of

387 individuals. In the event of clustering, we also propose a method to identify 'sub-

388 populations' of individuals that tend to associate during the two phases of their life cycle

389 (e.g. breeding and wintering).

390 The number of studies focusing on 'migratory connectivity' has been increasing

391 rapidly in recent years, mainly due to an expanding set of techniques, but also due to new

392 analyses of data from 'traditional' mark-recapture methods, allowing identification of

393 breeding and wintering areas of several bird species. To date, however, quantitative

394 methods to estimate migratory connectivity have been based on the calculation of origin

395 probability of individuals from a priori identified geographical populations or areas.

396 These methods do not test statistically for the deviation of the observed patterns of

397 reciprocal distribution of individuals from a random mix. The method we propose, which

398 is based on relatively simple calculations and statistics, may thus contribute to fill this

399 gap.

400 A prominent feature of our approach is that the investigation of migratory

401 connectivity is not based on an a priori identification of breeding and wintering 'sub- 
402 ranges', which may be difficult because several species show continuous distributions

403 both in the breeding and the wintering quarters. We propose that the identification of

404 highly connected ranges should be based on the actual distribution pattern of individuals.

405 This has the double advantage of reducing the subjectivity of the analysis and increasing

406 its biological realism by relying on inherent patterns present in the data.

407 In addition, we emphasize that our approach subtly differs from that based on the

408 a priori identification of separate sub-ranges. In fact, following that approach, the level of

409 connectivity would appear to differ depending on which of the different breeding and

410 wintering populations is currently under focus. Imagine, for example, a single group

411 (population) of individuals that breeds in an area (B) and winter in two well separated

412 areas (W1 and W2). B would be defined as moderately connected to either W1 or W2,

413 whereas both W1 and W2 would be defined as strongly connected to B. Thus, an

414 asymmetry is implicit in that approach, with a larger relevance usually given to the

415 breeding areas. Our approach solves this problem as equal relevance is given to each

416 distance matrix, and number and composition of groups of individuals is assessed $a$

417 posteriori by means of cluster analysis. In our approach, identification of sub-ranges and

418 highly connected areas follows from the identification of clusters of individuals that

419 connect regions by means of their migration.

420 Some of the features of our method deserve close consideration. First, the method

421 we used to combine the two distance matrices into the overall Euclidean distance matrix

422 implies that, for a given distance between two data-points in one range, their chances to

423 be classified in the same cluster declines as their distance in the other range increases.

424 This is desirable because, according to the symmetric approach that we are proposing 
425 (see above), only individuals that tend to both breed and winter together should be

426 classified into the same group. Second, the clustering procedure we chose involves the

427 comparison of the clustering efficiency, based on the oasw, when data are forced into 2 ,

$4283, \ldots, n$ clusters and the univocal identification of the best number of clusters. As a

429 drawback of the method, however, if the pattern of distribution of sampling locations

430 varies markedly between the breeding and the wintering areas, some clusters may be

431 obscured and forced by the clustering algorithm to join with other clusters. Finally, high

432 connectivity does not imply, per se, a highly clustered population. If, for example,

433 uniformly dispersed individuals in the breeding areas tend to maintain the same position,

434 relative to the other individuals, in the wintering areas (i.e. in the case of distribution

435 pattern transfer), the connectivity will be high even in the absence of a clear grouping of

436 individuals. Thus, our approach allows inferring connectivity arising from distribution

437 pattern transfer when the Mantel correlation coefficient is significant, but the whole

438 population cannot be partitioned into well-defined clusters.

439 The simulations we run under three different scenarios give further insights into

440 the interpretation of the results and the robustness of this method. First of all, the Mantel

441 correlation coefficient is able to detect connectivity due to distribution pattern

442 transference even when a large random noise is added to the data. This clearly emerges

443 from the results of the first and the third simulations. In the second and third simulation,

444 oasw values lower than the suggested threshold of 0.5 were recorded in several runs

445 where a cluster structure could then be identified, as indicated by the fact that the same

446 clustering algorithm was able to detect the correct number of clusters and to correctly

447 classify more than $95 \%$ of individuals in all but 18 simulations of the second and third 
448 type. In all cases where the method failed to detect connectivity, the distance between

449 cluster centres was not larger than $3 \mathrm{SD}$ in one area and not larger than $2 \mathrm{SD}$ in the other,

450 implying that the grouping structure was obscured by the fact that about $13 \%$ of

451 individuals mixed in one ground and about $60 \%$ in the other (details of this calculations

452 are not shown for brevity). On the other hand, Mantel correlation coefficients seemed

453 rather sensitive in detecting a structure in a population also when it arose from grouping

454 of individuals. Based on these results, we suggest to always investigate the cluster

455 structure of a population when the Mantel test is significant, and to use the value of the

456 Mantel correlation coefficient as a measure of migratory connectivity. In addition, we

457 tentatively suggest to investigate the structure of a group of individuals when the oasw is

458 larger than 0.4 , since, based on our simulations, the suggested threshold of 0.5 may be too

459 high. We admit, however, that further investigation is needed to identify an optimal

460 threshold for the oasw value.

461 Our method can be widely applied to different kinds of data about migratory birds

462 (and other migratory animals). Indeed, this method of analysis is applicable to any pair of

463 matrices of distance indices between individuals and not to geographical distances only.

464 For example, a large number of studies about migratory connectivity is based on the

465 analysis of the isotopic composition of feathers (see e.g. Hobson 2005). Differences in

466 the isotopic composition are considered to be related to the distance in the locations

467 where individuals moult their feathers at least in areas where specific geographical

468 gradients in isotopic abundance exist (Hobson 2005). Hence, a measure of the migratory

469 connectivity for a population can be obtained by correlating the matrix of geographic

470 distances between places where individuals were captured and the matrix of differences 
471 in the isotopic composition of their feathers. During moult, birds simultaneously wear

472 both recently moulted and old feathers when in the wintering or in the breeding areas.

473 The two matrices derived from isotopic profiles of newly moulted and old feathers should

474 be readily suitable for the analyses proposed here. Likewise, our method could be applied

475 to a matrix of isotopic profiles produced in winter and a second matrix of genetic markers

476 for the same individuals reflecting the population genetic structure of individuals at the

477 breeding grounds. We emphasize, however, that our method could be applied to distances

478 in the isotopic profiles and/or genetic distances provided that they are univocally related

479 to geographic distances between individuals. For example, deuterium values in North

480 America vary along a latitudinal gradient and were therefore used to study migration of

481 birds moving in a north-south direction (Hobson 2005), but this may not the case for

482 other areas or other isotopes, like ${ }^{13} \mathrm{C}$ and ${ }^{15} \mathrm{~N}$ in Africa, which provide habitat-specific,

483 rather than geographic area-specific markers (Møller and Hobson 2003), so that distance

484 in isotopic composition may not reflect true geographic distance between individuals. As

485 concerns genetic distances, these usually fulfil the criterion because isolation by distance

486 is a common feature of such distances.

487 We applied our method to a large ringing and recovery dataset of a Palearctic

488 migratory bird species that has been subject to intensive ringing programmes. We found

489 that the Western Palearctic breeding population of barn swallows is structured into two

490 main 'sub-populations'. The first population breeds in South-Western Europe and winters

491 in an elongated belt from Liberia to Uganda, while the second population breeds in

492 Northern Europe and winters south of the Equator. A second level of analysis showed

493 that both main groups could be split into two sub-groups. Barn swallows breeding in 
494 South-Western Europe could be divided according to segregation in the wintering

495 quarters with a first sub-group including barn swallows wintering from Liberia to

496 Cameroon and a second including barn swallows that winter from Cameroon to Uganda.

497 Conversely, the second main group can be divided according to segregation in the

498 breeding grounds, with a first sub-cluster that includes barn swallows mainly breeding in

499 North-Eastern Europe and a second group including barn swallows that breed in North-

500 Western Europe (Fig. 5b).

501 This pattern was identified based on the entire dataset (1103 individuals),

502 although the analyses were possibly confounded by the fact that either breeding and

503 wintering ringing or recovery could partly refer to migrating individuals. In addition,

504 differences in ringing effort at each ringing location may skew the results as the number

505 of ringed birds recovered at each location can be considered proportional to capture

506 effort. This problem could be exacerbated by the fact that larger ringing effort may be

507 devoted in areas with higher bird densities. Unfortunately, no quantitative measure of

508 ringing effort was available so it was impossible to correct for this potential bias.

509 However, the analyses based on different subsets of data identified according to

510 increasingly conservative criteria gave largely consistent results to those based on the

511 entire dataset, suggesting that the analysis was robust to the interference of different

512 potential sources of bias and even sample size. However, for several bird species less

513 than 200 recoveries may be available and small sample size may reduce the power of the

514 tests. Further theoretical as well as simulation studies are therefore needed to assess the

515 effect of sample size on the detection of migratory connectivity. However, some

516 preliminary results indicate that this method is robust when at least 35 recaptures are 
517 available (R. Ambrosini, unpublished results). In addition, we emphasize that our main

518 goal was to propose a method to quantify migratory connectivity, and that this large set of

519 real data was only used to exemplify its application.

520 Despite these potential drawbacks, the results of the present analysis of

521 connectivity can be qualitatively compared to the results obtained using different

522 approaches. The subdivision of the Western Palearctic barn swallow population in two

523 main sub-populations is roughly consistent with the observation of differences in the

524 quantitative genetic variance-covariance matrix (G-matrix) between barn swallows from

525 Northern and Southern Europe (Roff et al 2004). In addition, in an analysis of the isotopic

526 composition of feathers, Evans et al (2003) identified segregation in the wintering

527 quarters of barn swallows breeding in England and Switzerland. This is consistent with

528 our results, as British barn swallows are mainly classified in a group that winters in

529 Southern Africa, while Swiss barn swallows were included in the cluster wintering north

530 of the Equator. Moreover, stable isotope composition of feathers of barn swallows

531 indicates that birds that breed in Denmark winter in at least two different areas with

532 different levels of $\delta^{15} \mathrm{~N}$ (Møller and Hobson 2003). This is consistent with our results,

533 since barn swallows breeding in Denmark were classified partly in the first and partly in

534 the second first-level clusters, whose African wintering grounds are separated. High $\delta^{15} \mathrm{~N}$

535 values in willow warbler (Phylloscopus trochilus L) feathers sampled in South Africa

536 was reported by Bensch et al. (2006). If we assume that isotopic profiles in barn swallow

537 feathers are not dissimilar to those in the willow warbler, we can go further and

538 tentatively suggest that $\delta^{15} \mathrm{~N}$ enriched cluster of Møller and Hobson should correspond to

539 cluster All.2 and the $\delta^{15} \mathrm{~N}$ depleted one to cluster All.1. 
These results suggest that analyses of stable isotopes or other markers of group

541 identity of barn swallows should provide evidence of clusters similar to those reported in

542 Fig. 4, both when based on captures in the winter quarters in Africa or at the breeding

543 grounds in Europe. Hence, the connectivity pattern we found based on ringing recoveries

544 is largely consistent with the picture provided by studies of individual breeding

545 populations based on different approaches. In addition, although the identification of a

546 structure of the barn swallow population under focus is not novel, our analysis provides a

547 first quantitative measure of migratory connectivity.

548 In conclusion, we have proposed a quantitative measure of migratory connectivity

549 and a method for identification of 'sub-populations' that are amenable to inferential

550 statistical analysis and open the possibility of quantitatively inyestigating the

551 relationships between different areas due to the movement of migrants between them.

552 This measure can be quickly calculated for several species as large sets of data from

553 recoveries are already available at least for the most common species, and datasets on

554 genetic and isotopic distances between populations are rapidly growing. In addition, a

555 quantitative measure of migratory connectivity allows the comparison of migration

556 strategies of different species and/or populations and thus allows further investigation of

557 the ecological bases of the evolution of migratory systems. Finally, this method allows

558 estimates of migratory connectivity between geographic regions based on assemblages of

559 species that migrate between them. Such a measure may provide a substantial

560 contribution in the planning of effective conservation strategies for migratory species. 


\section{Acknowledgments}

562

563 EURING, SAFRING and numerous ringing centres in Europe kindly provided access to

564 their recoveries of ringed barn swallows. We are grateful to Dr. Riccardo Borgoni and to

565 two anonymous referees whose comments greatly improved the quality of the

566 manuscript.

567 
569 Anderson, Á., Follestad, A., Nilsson, L., Persson, H., 2001. Migration patterns of Nordic

$570 \quad$ Greylag Geese Anser anser. Ornis Sve. 11, 19-58.

571

572 Besag, J., Diggle, P. J., 1977. Simple Monte Carlo tests for spatial pattern. Appl. Stat. 26, $573 \quad 327-333$.

575 Bensch, S., Bengtsson, G., Åkesson, S., 2006. Patterns of stable isotope signatures in 576 willow warbler feathers collected in Africa. J Avian Biol, 37, 323-330., doi:

$577 \quad \underline{10.1111 / \mathrm{j} .2006 .0908-8857.03628 . \mathrm{x}}$

579 Cohn, J. P., 1999. Tracking wildlife: high-tech devices help biologists trace the

580 movement of animals though sky and sea. BioScience 49, 12-17.

582 Evans, K. L., Waldron, S., Bradbury, R. B., 2003. Segregation in African wintering 583 ranges of England and Swiss Swallow Hirundo rustica populations: a stable isotope 584 study. Bird Study 50, 294-299.

586 Hobson, K. A., Wassenaar, L. I., 1997. Linking breeding and wintering grounds of 587 Neotropical migrant songbirds using stable hydrogen isotopic analysis of feathers.

588 Oecologia 109, 142-148., doi: 10.1007/s004420050068 
590 Hobson, K. A., 2005. Stable isotopes and the determination of avian migratory

591 connectivity and seasonal interactions. Auk 122, 1037-1048.

592

593 Hooge, P. N., Eichenlaub, B., 2000. Animal movement extension to Arcview. Ver. 2.0. -

594 Alaska Science Centre - Biological Science Office, U.S. Geological Survey, Anchorage,

595 AK, USA. Available from: http://www.absc.usgs.gov/glba/gistools/index.htm (accessed

596 July 2007)

597

598 Jennrich, R. I., Turner, F. B., 1969. Measurement of non-circular home range. J. Theor.

599 Biol. 22, 227-237.

600

601 Kaufman, L., Rousseeuw, P. J., 1990. Finding Groups in Data: An Introduction to Cluster

602 Analysis. Wiley, New York.

603

604 Lopes, R. J., Marques, J. C., Wennerberg, L., 2006. Migratory connectivity and temporal

605 segregation of dunlin (Calidris alpina) in Portugal: evidence form morphology, ringing

606 recoveries and mtDNA. J, Ornithol. 147, 85-94. 10.007/s10336-005-0048-y.

607

608 Marra, P. P., Hobson, K.A., Holmes, R.T., 1998. Linking winter and summer events in a

609 migratory bird by using a stable-carbon isotopes. Science 288, 2040-2042., doi:

$610 \quad 10.1126 /$ science.282.5395.1884

611

612 Marra, P.P., Norris, D.R., Haig, S.M., Webster, M., Royle, J.A., 2006. Migratory 
613 connectivity. In: Crooks, K. R., Sanjan, M. (Eds) Connectivity Conservation. Cambridge

614 University Press, Cambridge, pp 157-183.

615

616 Marshak, S., 2001. Earth: Portrait of a Planet. W. W. Norton \& Company, New York.

617

618 Møller, A. P., Hobson, K.A., 2003. Heterogeneity in stable isotopes profiles predicts

619 coexistence of populations of barn swallows Hirundo rustica differing in morphology and

620 reproductive performance. Proc. R. Soc. B. 71, 1355-1362., doi: 10.1098/rspb.2003.2565.

621

622 Roff, D. A., Mousseau, T., Møller, A. P., de Lope, F., Saino, N., 2004. Geographic

623 variation in the $\mathrm{G}$ matrices of wild populations of barn swallow. Heredity 93, 8-14., doi:

$624 \quad 10.1038 /$ sj.hdy.6800404

625

626 Rousseeuw, P. J., 1987. Silhouettes: A graphical aid to the interpretation and validation

627 of cluster analysis. J. Comput. App. Math. 20, 53-65.

628

629 Salomonsen, F., 1955. The evolutionary significance of bird migration. Biologiske

630 Meddelelser 22, 1-62.

631

632 Sokal, R. R., Rohlf, F. J., 1995. Biometry: The Principles and Practice of Statistics in

633 Biological Research, 3rd edn. Freeman, New York

634

635 Szép, T., Møller, A. P., Piper. S., Nuttall, R., Szabó, Z. D., Pap, P. L., 2006. Searching for 
636 potential wintering and migration areas of a Danish Barn Swallow population in South

637 Africa by correlating NDVI with survival estimates. J. Ornithol. 147, 245-253., doi:

$638 \quad 10.1007 / \mathrm{s} 10336-006-0060-\mathrm{x}$

639

640 Turner, A. K., 1994. The swallow. Hamlyn, London.

641

642 Webster, M. S., Marra, P. P., Haig, S. M., Bensch, S., Holmes, R. T., 2002. Links

643 between worlds: unravelling migratory connectivity. Trends Ecol. Evol. 17, 76-83., doi:

$644 \quad 10.106 / \mathrm{S} 0169-5347(01) 02380-1$.

645 
647 Figure 1. Calculation of orthodromic distance between points A and B along a spherical 648 surface. Geographical coordinates (latitude $=\varphi$ and longitude $=\lambda$ ) are first converted

649 into three-dimensional Cartesian coordinates $(x, y, z)$ with Eq. 1-3 in figure. $r$ is the

650 average radius of the Earth $(6371 \mathrm{~km}$; Marshak 2001). Euclidean distance $(d)$ between the

651 points is easily calculated from Cartesian coordinates by means of Pythagoras' theorem

652 and then converted into orthodromic distance $(l)$ with Eq. 4.

653

654 Figure 2. Results from the second simulation. Oasw: overall average silhouette width.

655 Gray surface colour indicates a simulation with a significant $(P \leq 0.05)$ Mantel

656 correlation coefficient. Black surface colour represents non-significant simulations. Plans

657 represent oasw values of 0.5 and 0.7 respectively. Distances between cluster centres are

658 expressed as standard deviations (SD) of the bivariate Gaussian used to generate

659 individual position within clusters.

660

661 Figure 3. Dendrogram of the analysis (a) of the whole set of recoveries and recaptures of

662 barn swallows (All) and (b) of the subset of data only including barn swallows ringed in

663 Europe as adults (AE). $r_{M}$ : Mantel correlation coefficient; $P$ : significance of the Mantel

664 test as assessed with the randomization procedure; oasw: overall average silhouette

665 width.

666

667 Figure 4. First level clusters for different selection criteria. a) All barn swallows . b) 
668 Barn swallows recorded in the breeding and wintering grounds in the focal periods of

669 reproduction and wintering respectively. c) Barn swallows ringed in Europe as adults. d)

670 Barn swallows ringed in Europe as adults in the focal period of reproduction and

671 recovered in Africa in the focal period of wintering.

672

673 Figure 5. Pictorial representation of first (a) and second (b) level clusters. Ellipses

674 represent the Jennrich and Turner (1969) Bivariate Normal Home Range calculated with

675 the Animal Movement extension to ArcView Gis (Hooge and Eichenlaub 2000). Dashed

676 ellipses in (a) represents the simulated position of cluster All.2 used to assess the

677 robustness of the method with a less geographically structured dataset.

678

679 Figure 6. Silhouette plot showing the classification of the All dataset in two first-level

680 clusters. Each bar represents the silhouette values $\mathrm{s}(i)$ for a single barn swallow (see also

681 Statistical methods). Within each cluster, bars are drawn in decreasing length order.

682 Large values indicate good classification.

683 
Figure 1

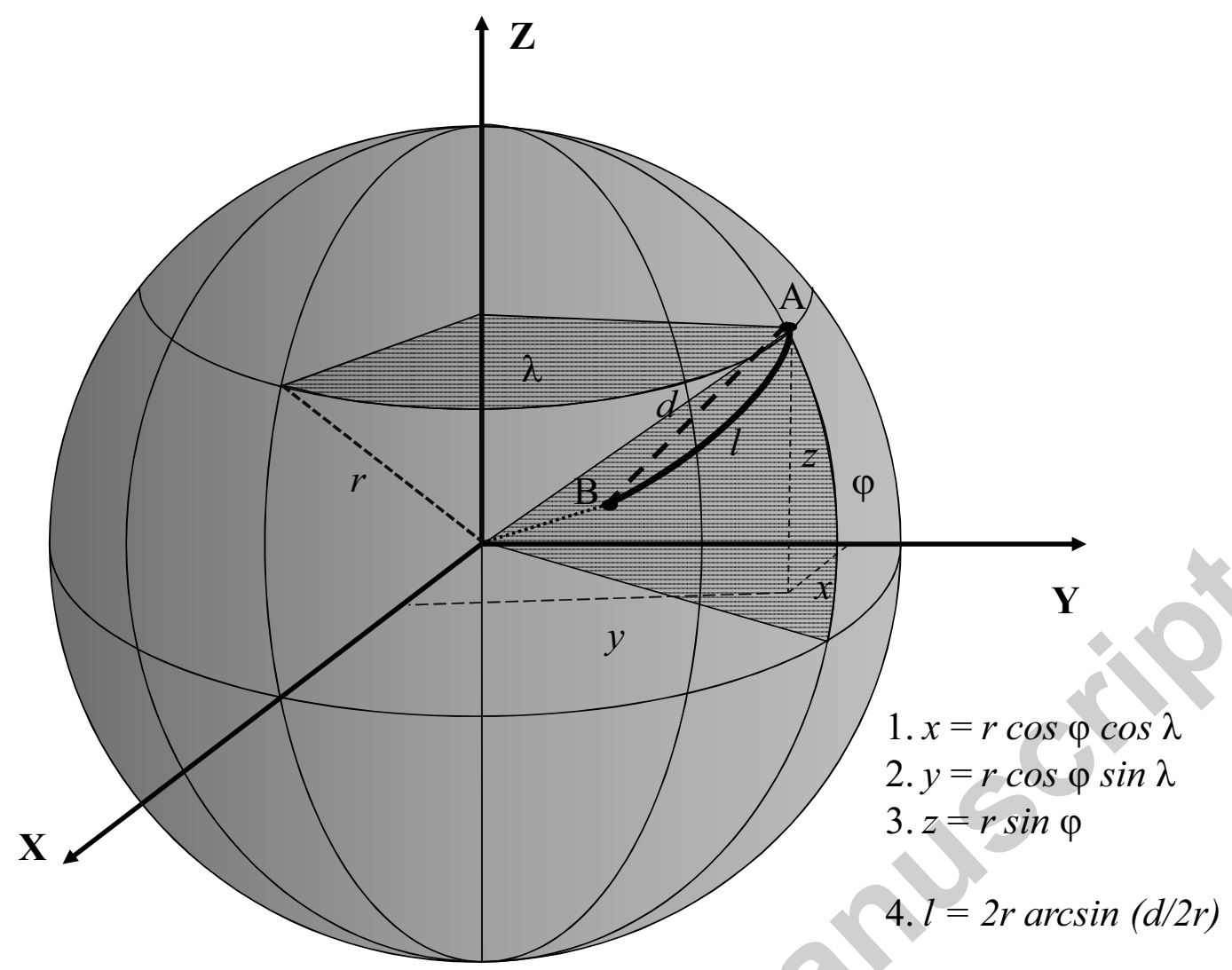




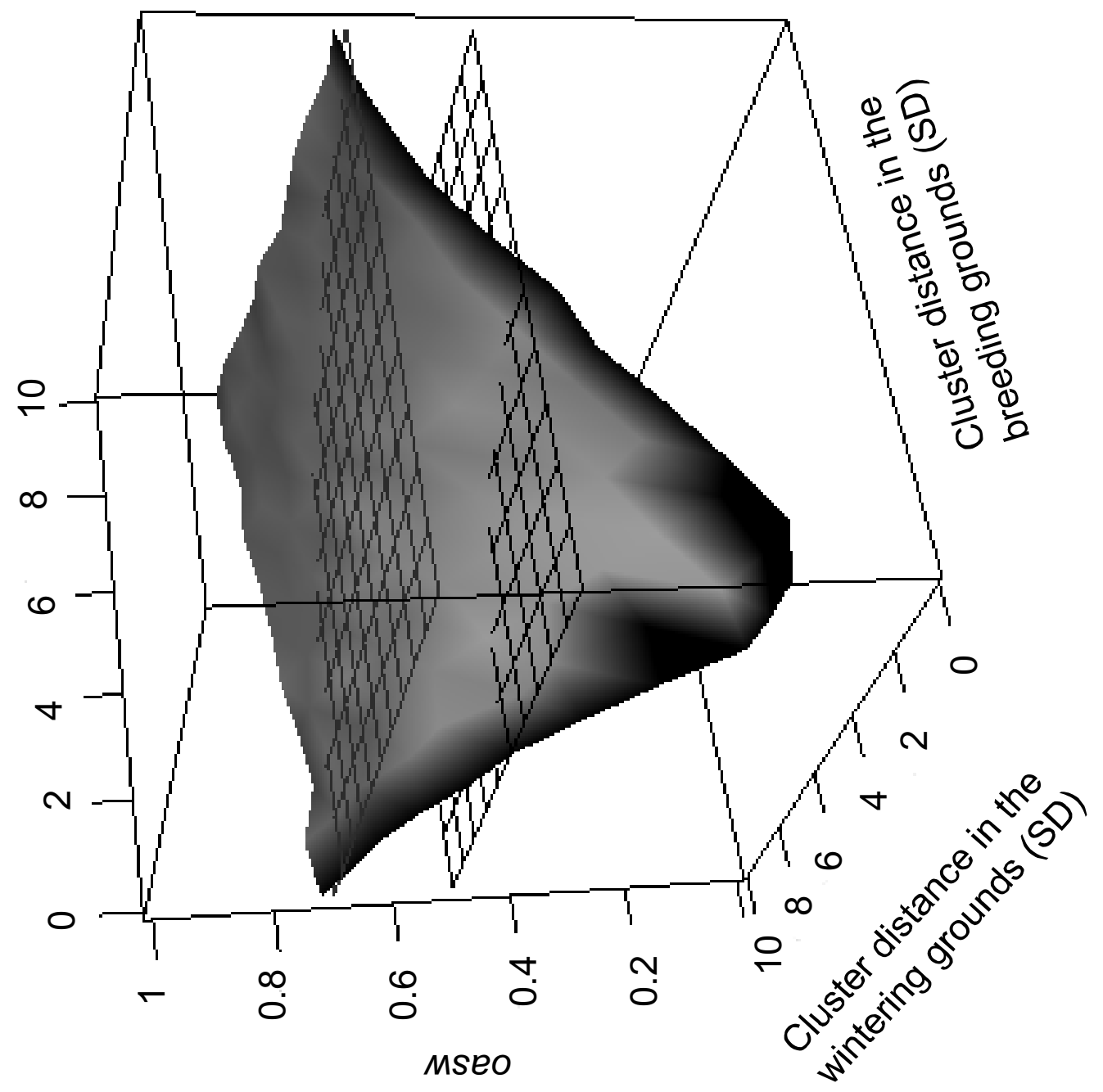

N
ํㅣㄴ
은 
Figure 3

a

All (n= 1103)
$r_{M}=0.0247$
$P=0.054$
oasw $=0.610$

\begin{tabular}{|c|c|c|c|}
\hline $\begin{array}{l}\text { All.1 }(\mathbf{n}=\mathbf{1 4 1}) \\
r_{M}=0.0644 \\
P=0.055 \\
\text { oasw }=0.433\end{array}$ & $\begin{array}{l}\text { 41) } \\
3\end{array}$ & \multicolumn{2}{|c|}{$\begin{array}{l}\text { All } .2(\mathbf{n}=\mathbf{9 6 2}) \\
r_{M}=0.0593 \\
P<0.001 \\
\text { oas } w=0.553\end{array}$} \\
\hline $\begin{array}{l}\text { All.1.1 }(\mathbf{n}=\mathbf{9 6}) \\
r_{M}=0.0175 \\
P=0.292\end{array}$ & $\begin{array}{l}\text { All.1.2 }(\mathbf{n}=\mathbf{4 5}) \\
r_{M}=0.2381 \\
P=0.004\end{array}$ & $\begin{array}{l}\text { All .2.1 }(\mathbf{n}=\mathbf{3 8 8}) \\
r_{M}=0.0804 \\
P=0.002\end{array}$ & $\begin{array}{l}\text { All.2.2 }(\mathbf{n}=\mathbf{5 7 4}) \\
r_{M}=0.0251 \\
P=0.181\end{array}$ \\
\hline
\end{tabular}

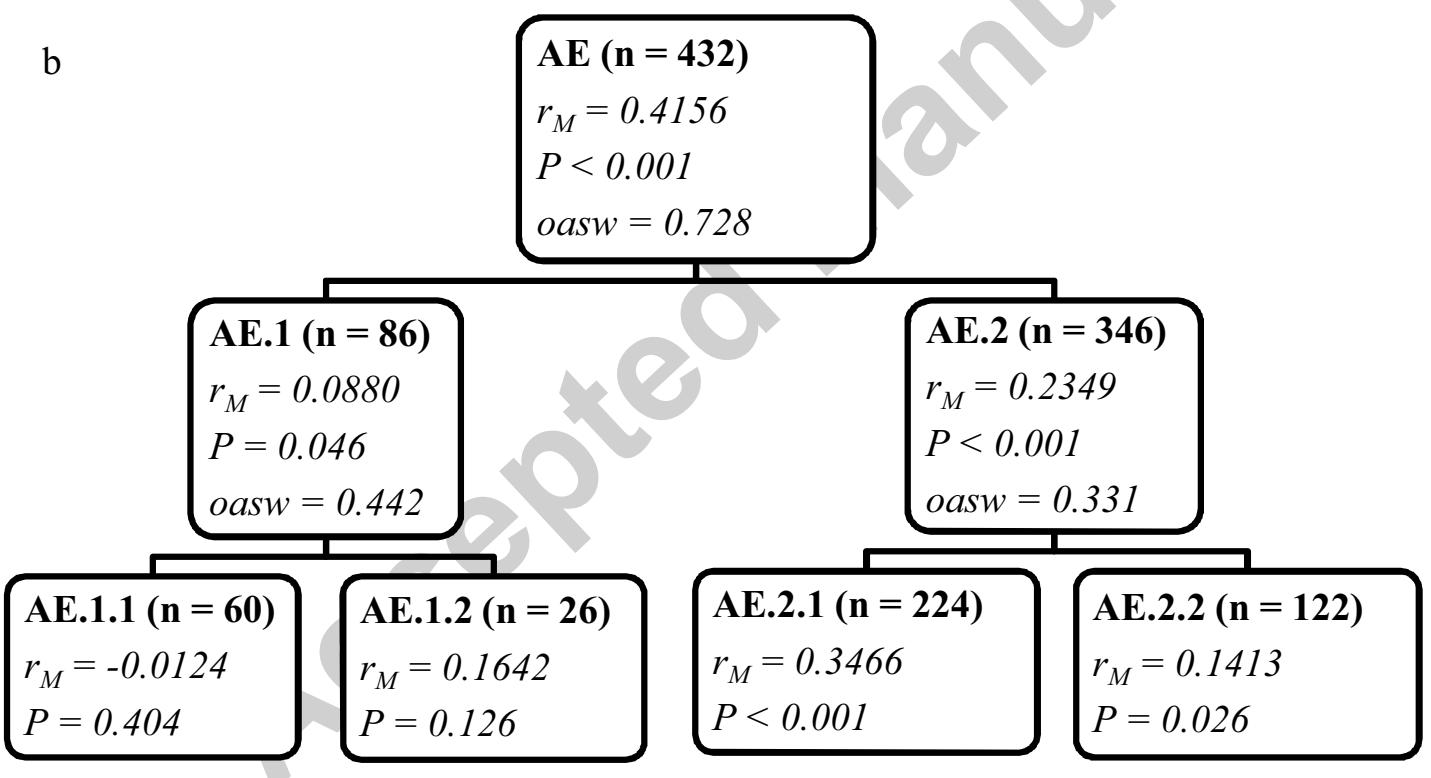




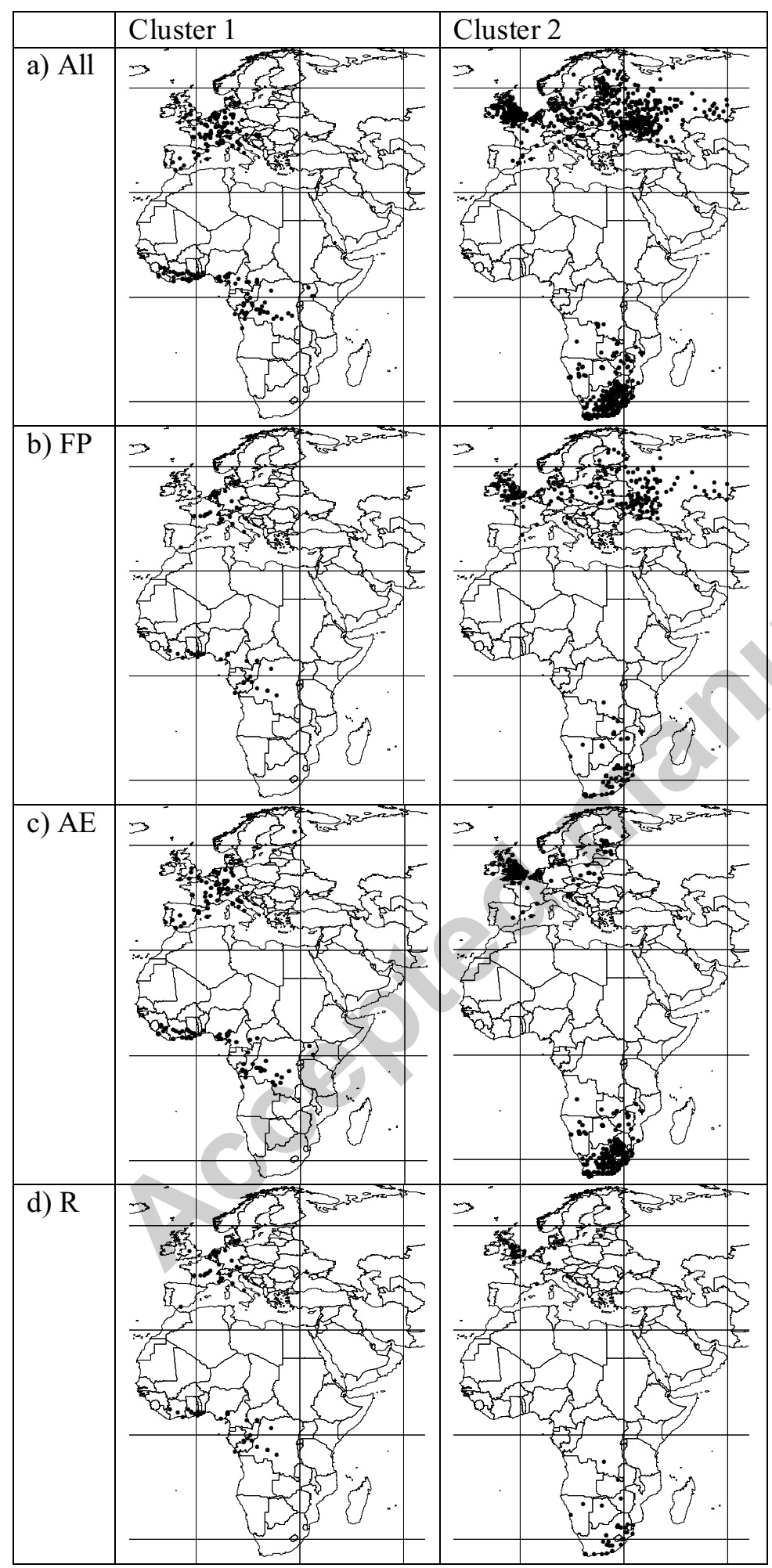


Figure 5

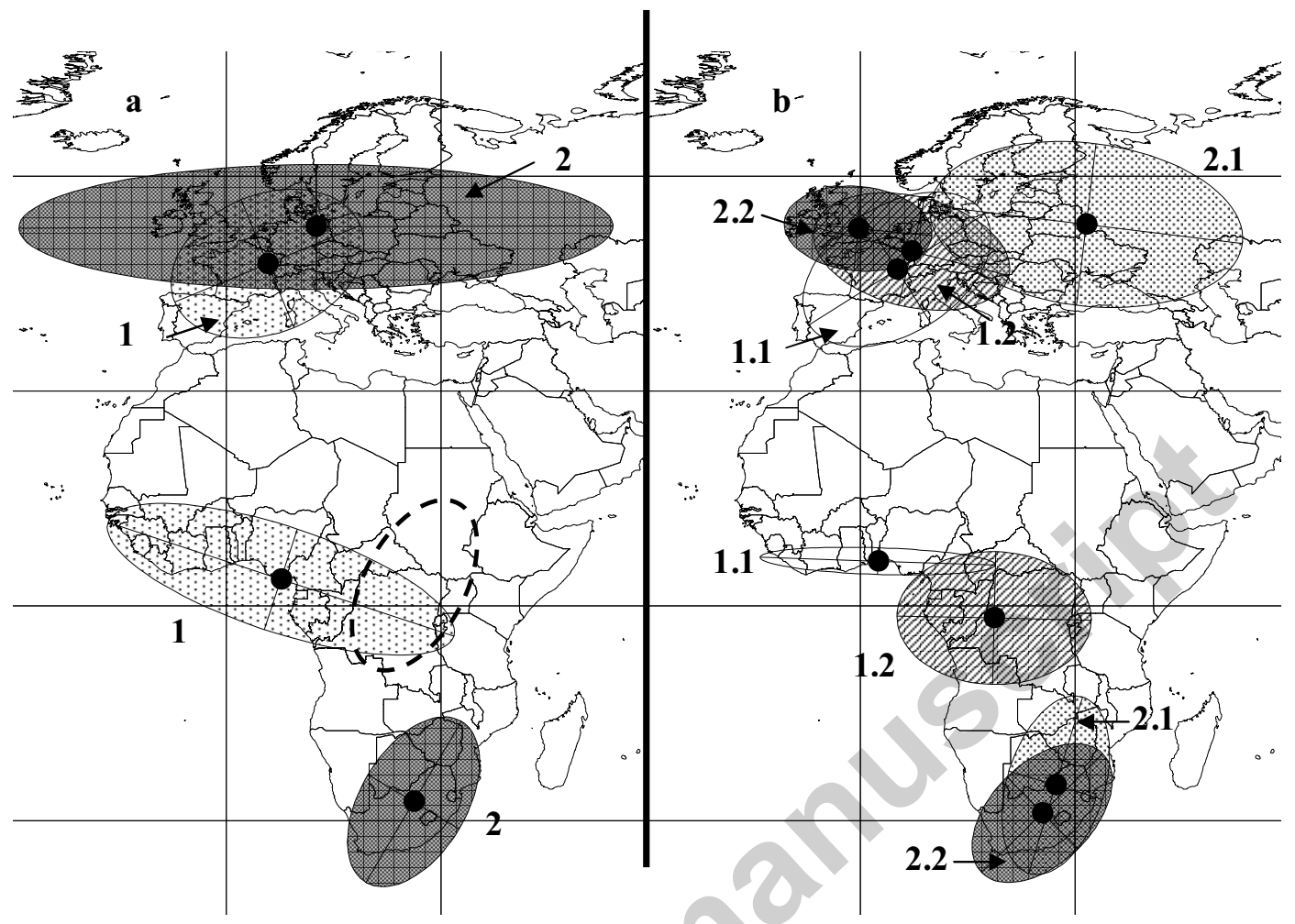




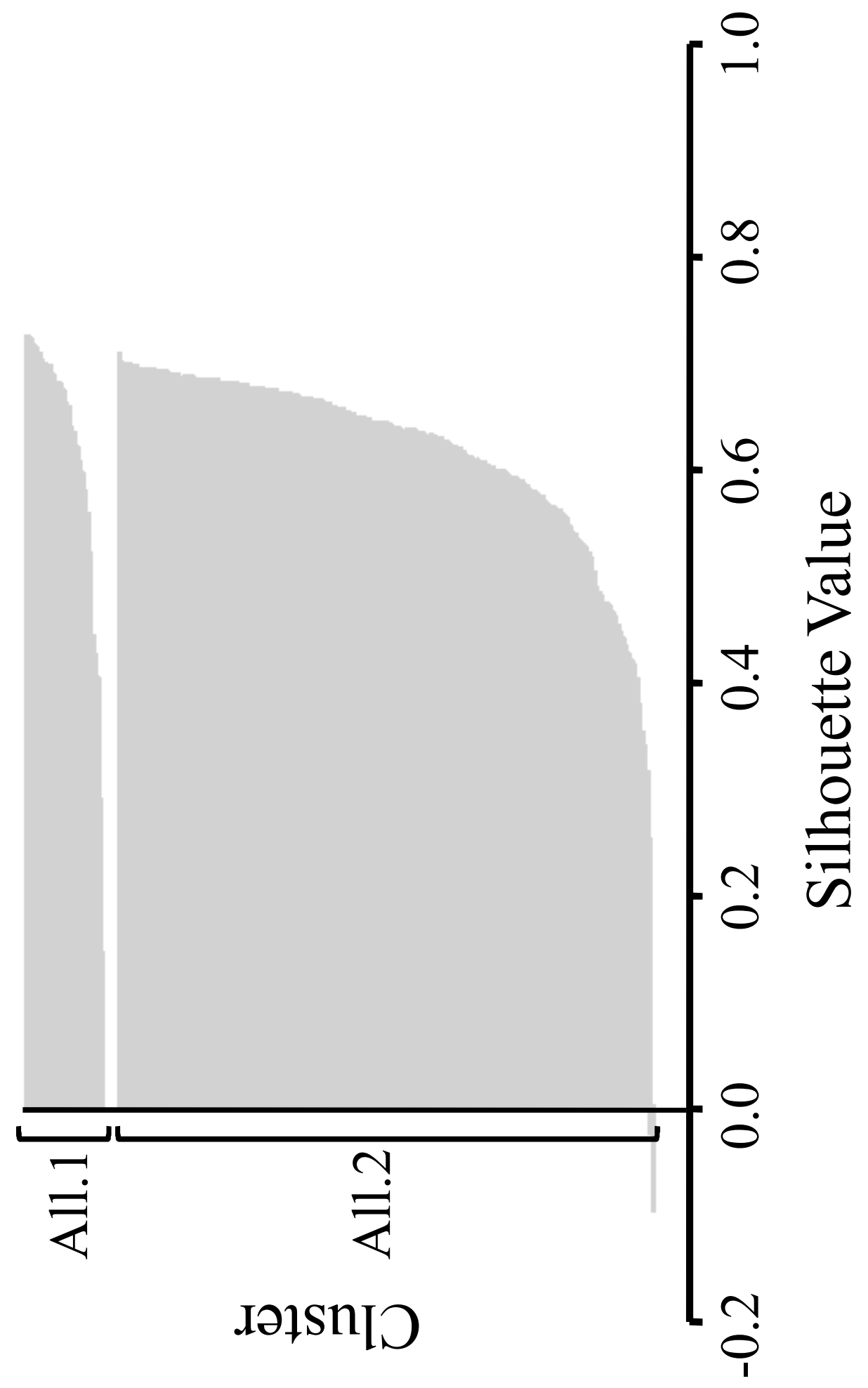

